\title{
Downlink data rate, energy and spectral efficiency distribution in heterogeneous networks with cell-edge located small cells
}

\author{
Güven Yenihayat ${ }^{1}$ (D) Ezhan Karaşan ${ }^{1}$
}

Published online: 20 May 2019

(C) Springer Science+Business Media, LLC, part of Springer Nature 2019

\begin{abstract}
In this paper, the probability distributions of per user downlink data rate, spectral efficiency (SE) and energy efficiency (EE) are analytically derived for a heterogeneous network model with cell-edge located small cells. The high accuracies of analytically derived cumulative distribution functions (CDF) are verified using distributions obtained via simulations. CDF expressions are then used in order to optimize key performance indicators (KPI) which are selected here as 10th percentile downlink data rate $\left(R_{10}\right)$, spectral efficiency $\left(S E_{10}\right)$ and energy efficiency $\left(E E_{10}\right)$. In addition to optimizing KPIs separately, we also investigate the variation of the KPIs with respect to each other employing the analytically derived distributions. The results show that the resource allocation parameter values maximizing $R_{10}$ are very close to the values that maximize $E E_{10}$. However, the values that are optimal for $E E_{10}$ and $R_{10}$ are not optimal for $S E_{10}$, which demonstrates the EE and SE trade-off in HetNets.
\end{abstract}

Keywords Heterogeneous networks - Downlink data rate distribution - Spectral efficiency/energy efficiency trade-off

\section{Introduction}

With the ongoing evolution of mobile devices, the demand for higher data rates in mobile communication systems has been increasing rapidly. According to the Wireless World Research Forum's (WWRF) vision for 2020, a mobile traffic growth of 1000 times compared to current generation of wireless standards is expected [1]. According to $5 \mathrm{G}$ visions of ITU and several communication companies, the services in $5 \mathrm{G}$ will require higher data rates, lower latency and higher reliability. All these improvements should be done in a cost effective manner [2]. In order to satisfy the 1000x data challenge, the key technological targets are increased bandwidth, increased spectral efficiency and extreme cell densification [3, 4].

Cell densification is a key enabler for $5 \mathrm{G}$ networks $[3,5]$. By shrinking the cell sizes, the spectrum can be

\section{Güven Yenihayat}

guven@ee.bilkent.edu.tr

Ezhan Karaşan

ezhan@ee.bilkent.edu.tr

1 Department of Electrical and Electronics Engineering, Bilkent University, Ankara, Turkey reused across the area which increases the per user rates. In dense deployments, adding more base stations (BS) also increases the interference levels. In order to overcome this problem, deployment of BSs with lower transmit power is proposed. Low power base stations are named as micro, pico and femto base stations depending on their transmit powers. Networks consisting of a mix of these base stations are called Heterogeneous Networks (HetNets) [5, 6]. In HetNets, with the addition of small cells, the area spectral efficiency is increased. For the ongoing 3GPP development, there are various scenarios and requirements for the enhancement of small cells [7]. As in [8], cell range expansion (CRE) is one of the methods in HetNets to offload more users to small cells, which is enabled through cell biasing and adaptive resource partitioning. CRE is seen as an effective method to balance the load among the nodes in the network and to improve overall trunking efficiency. Although the received power from Macro cell is larger with cell biasing, the UE associates itself with a small cell as long as the difference between the received powers from the macro cell and small cell is smaller than the positive bias value. With cell biasing, depending on the bias value, the network can control the number of user equipments (UE) associated with the low-power nodes and therefore 
offload more traffic demand to those nodes [5]. In this study, we assume a two-tier HetNet, where there are Macro and Micro BSs that use cell biasing. In the considered scenario, there are three types of UEs: UEs associated with the Macro BS are named as Macro UEs, UEs associated with a Micro BS with zero biasing are called Direct Micro UEs, and UEs associated with a Micro BS with positive biasing are called CRE UEs.

In order to minimize the interference among the users of the system, time/frequency resources should be partitioned carefully in HetNets. For instance, when CRE UEs and Macro UEs are being served in the same time interval and at the same frequency band, the received signal power of CRE UEs will be lower than the interference power coming from the Macro cell. Therefore, the resources are required to be orthogonally shared between CRE UEs and Macro UEs. This can be done by using the Almost Blank Subframe (ABS) technique which is a part of the Enhanced Intercell Interference Coordination (eICIC) developed by 3 GPP working group [9]. As stated in [9], in ABS, Macro BS does not transmit while CRE UEs are being served, so that CRE UEs do not suffer from the Macro BS interference. The resource allocation between Direct Micro UEs and Macro UEs can be done by using orthogonal or nonorthogonal deployments [10]. In this study, we assume orthogonal deployment between Direct Micro and Macro UEs so that Macro BS interference at Direct Micro UEs and Micro BS interference at Macro UEs are eliminated.

Throughout this work, we assume Cell-On-Edge (COE) configuration as the HetNet model and use a resource allocation scheme which partitions the resources in time and frequency. We analytically derive the probability distribution of the downlink data rates achieved by users and then verify the proposed analytical model by simulations. We assume that orthogonal frequency planning is done among neighboring Micro BS cells so that no interference is coming from neighboring cells. We also assume that Macro and Micro BSs always transmit with constant power, i.e., no power control is done. We show that the distributions obtained from the analytical model are highly accurate under a wide range set of network parameters such as spatial user distribution and bias. By using the analytical rate distribution, we optimize the system in terms of 10th percentile rate $\left(R_{10}\right)$, which corresponds to the $10 \%$ point of the cumulative distribution function (CDF) of the per user data rate. In addition to the user rate, energy and spectral efficiencies are other key performance metrics in $5 \mathrm{G}$ networks. We also derive the CDF expressions for the Spectral Efficiency (SE) and Energy Efficiency (EE), and then optimize the resource allocation parameters in order to maximize the tenth percentile $\mathrm{SE}\left(S E_{10}\right)$ and $\mathrm{EE}\left(E E_{10}\right)$. The results reported in the paper demonstrate the SE and EE trade-off in the studied HetNet model, and the analytically obtained distributions can be used to address the SE-EE trade-off according the preferences of the network service provider.

The most important contributions of this paper are:

- Using a fully analytical approach, CDF of downlink data rate per user, SE and EE are derived for a HetNet with COE configuration. The analytically derived CDFs have been shown to be very close to CDFs obtained from simulations under an extensive set of spatial user distributions and bias values.

- Using analytically obtained distributions, optimal resource allocation parameters are calculated that maximize $R_{10}, S E_{10}$ and $E E_{10}$.

- Our results show that the optimal values of resource allocation parameters maximizing $R_{10}$ and $E E_{10}$ are close to each other, however these values are not optimal for $S E_{10}$.

The rest of this paper is organized as follows: Sect. 2 gives relevant studies in the literature. Section 3 describes our system model for the HetNet in consideration. Section 4 presents the derivation of the cumulative distribution function of the rate/user, spectral efficiency and energy efficiency for the network model and the resource allocation scheme employed. Section 5 presents the simulation and analytical results and the paper is concluded in Sect. 6 .

\section{Related work}

The distributions of SINR and user rate are crucial parameters for system optimization in wireless networks. Stochastic geometry (SG) and hexagonal grid structure based models have been used to obtain the analytical expressions for distributions of SINR, user rate and coverage in wireless networks [11]. For multi-tier cellular networks different types of point processes have been employed. For example, in [12-18], Poisson-Point-Process (PPP) based models are used to investigate the multi-tier cellular networks in terms of coverage probability and ergodic rate. Another way of modeling HetNets is using static models. One of the static models which is appropriate for practical deployments is the Cell-on-edge (COE) configuration [19]. COE configuration is a practical model in which the macro BS is located in the center and small cells are placed regularly around a ring that is close to the cell boundary. COE configuration has been shown to produce significant benefits in terms of improved cell-edge coverage, increased network capacity, enhanced end-user experience, and reduced cost of delivering mobile broadband services to cell-edge mobile users [19]. COE deployment model is employed in this study. 
We obtain the distributions of rate, spectral and energy efficiency and use them to optimize the cell range expansion bias and resource allocation parameters. There are several studies in the literature investigating the optimal selection of resource allocation parameters, range expansion bias values and user associations such as [20-25]. The HetNet models used in these papers are similar to the HetNet deployment we use in this study. In [20], similar to our study the distribution of SINR of users is obtained to evaluate the benefits of range expansion in HetNets. However different than our study, co-channel deployment among users in different tiers is assumed, and the distribution of SINR is obtained by sweeping user locations on grid points using simulations. In our study we obtain the CDFs of rate, spectral and energy efficiency fully analytically. In [21], optimal CRE bias values are obtained using simulations to maximize the sum rate. In our study, by using the analytically derived data rate $\mathrm{CDF}$, we optimize the system in terms of tenth percentile rate which also considers the fairness whereas maximizing the sum rate does not take fairness into account. In [22], instantaneous CDF of SINR is used to dynamically optimize the bias and time resource sharing parameter. The benefits of the proposed method on throughput is investigated by simulations. Instead of using an analytical model in order to obtain SINR CDF as we have done in this paper, [22] assumes that pico BSs collect the real SINRs of the users and use these values to calculate the optimal system parameters. In $[23,24]$, several radio resource management and interference coordination schemes with various CRE bias values are evaluated via simulations for a HetNet model. In [25], the optimal time resource allocation parameter selection problem is formulated as a mixed integer nonlinear programming problem, and the problem is solved in order to maximize the user with the minimum data rate. Maximizing the minimum data rate in the network may generate deceptive results when a user has a significantly inferior channel to the BS. Instead, we use the group of users with the lowest data rates, e.g., $10 \%$, to measure the fairness of the data rate distribution in the network as a performance indicator in this paper. Analytically obtained CDFs of rate, spectral and energy efficiencies can also be used in order to optimize other performance metrics that can be defined by the network operator.

\section{System model}

We consider a heterogeneous network model which consists of Macro and Micro Base Stations (BS) and User Equipments (UE). We use the Cell-On-Edge Model where Micro BSs are located on the edge of a Macro cell. The model follows the assumptions, which are in accordance with the 3GPP model given in [26]:

- There is one Macro BS located at the center of a circular area with radius $L$.

- There are $N_{\text {MICRO }}$ Micro BSs that are located on the ring that is located $d_{\text {Micro }}$ away from the center. The distances between adjacent Micro BSs are equal.

- A portion of the user equipments (UEs) are uniformly distributed over the entire area.

- The remaining UEs are located uniformly within circles that are in the coverage of Micro BSs. The ratio of the number of these UEs to all UEs is $W_{\text {Micro }}$.

One example topology is shown in Fig. 1, where denser distribution of users around micro base stations can be observed. As $W_{\text {Micro }}$ increases, the density around Micro BSs increases.

In the communication system model, we only consider the downlink communication from the BSs to UEs and assume that UEs have always something to receive from BSs (saturated traffic model). The wireless channel between BSs and UEs is modeled by a path loss model for which the received power $\left(P_{r, i}\right.$ in Watts $)$ is related to the transmit power $\left(P_{t, i}\right.$ in Watts) of BS with index $i$ as in

$P_{r, i}=\frac{P_{t, i}}{d_{i}^{\gamma_{i}}}$

where $\gamma_{i}$ is the path loss exponent and $d_{i}$ is the distance between BS $i$ and UE. The path loss model given in (1) omits channel impairments such as Rayleigh fading and shadowing. During network planning, resource allocation parameter optimization uses average received powers that primarily depend on the distance between BS and UEs. The

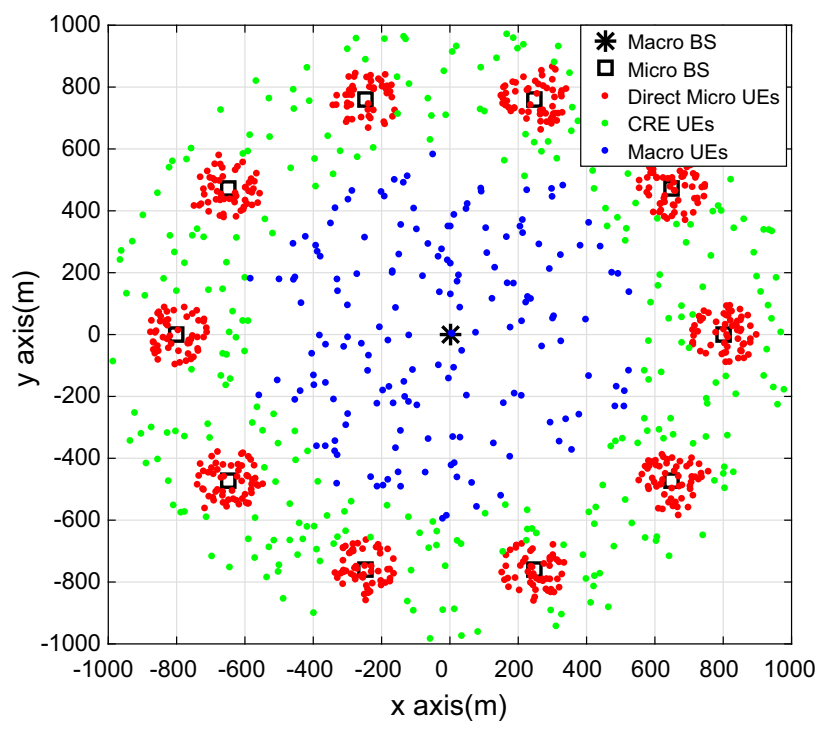

Fig. 1 An example of topology and UE connections for $B=15 \mathrm{~dB}$ 
optimized parameter values during network planning may not be optimal during network operation due to varying channel conditions, but these values can be used as initial parameter values that can be further tuned during networks operations using the real-life channel measurements collected while the network is operational. Throughout the paper, we use the following convention for BS type to index mapping: the $\mathrm{BS}$ with index $i=0$, i.e., $B S_{0}$, is the Macro BS and the BSs with index $i>0$ are Micro BSs. Consecutive index numbers correspond to neighbor Micro BSs, for example $B S_{1}$ and $B S_{2}$ are neighbors. And also due to circular placement of Micro BSs, $B S_{1}$ and $B S_{N_{\text {MICRO }}}$ are also neighbors. The path loss exponent $\gamma_{i}$, between UE and $B S_{i}$ is given as

$\gamma_{i}=\left\{\begin{array}{ll}\alpha_{1}, & \text { if } \quad i=0 \\ \alpha_{2}, & \text { if } i>0\end{array}\right.$.

where $\alpha_{2}>\alpha_{1}$ due to lower heights of Micro BSs. In (1), the transmit power $P_{t, i}$ differs depending on the BS type and it is given as

$P_{t, i}=\left\{\begin{array}{ll}P_{1}, & \text { if } \quad i=0 \\ P_{2}, & \text { if } i>0\end{array}\right.$,

where $P_{1}>P_{2}$. In this system, each UE calculates its signal power parameter $P_{s, i}$, which is a scaled version of the received power $P_{r, i}$ with the bias value of $B S_{i}\left(B_{i}\right)$. Depending on the $P_{s, i}$, UE associates itself with a BS. The relation between $P_{s, i}$ and $P_{r, i}$ is given by $P_{s, i}=P_{r, i} 10^{\frac{B_{i}}{10}}$, where

$B_{i}=\left\{\begin{array}{ll}0, & \text { if } \quad i=0 \\ B, & \text { if } \quad i>0\end{array}\right.$.

UE is associated with $B S_{i}$ for which signal power parameter, $P_{s, i}$, is maximum. In the system, each UE can be a Macro, Cell Range Extended (CRE) or a Direct Micro UE. The UEs for which $P_{s, i}$ is maximum for $i=0$ are Macro UEs. The other UEs are either CRE or Direct Micro UEs depending on their received power parameter, $P_{r, i}$. Among the UEs whose $P_{s, i}$ is maximum for some $i>0$, the UEs whose $P_{r, i}$ is maximum for some $i>0$ are Direct Micro UEs, and the UEs whose $P_{r, i}$ is maximum for $i=0$ are CRE UEs. Figure 1 shows how UEs are associated with BSs for $B=15 \mathrm{~dB}$.

In our system, we use a resource allocation scheme which is shown in Fig. 2. According to this resource allocation scheme, CRE UEs are served in $\eta$ of time for $0 \leq \eta \leq 1$, whereas Direct Micro UEs and Macro UEs are served in the remaining $1-\eta$ amount of time. In addition to partitioning in time domain, we also employ a

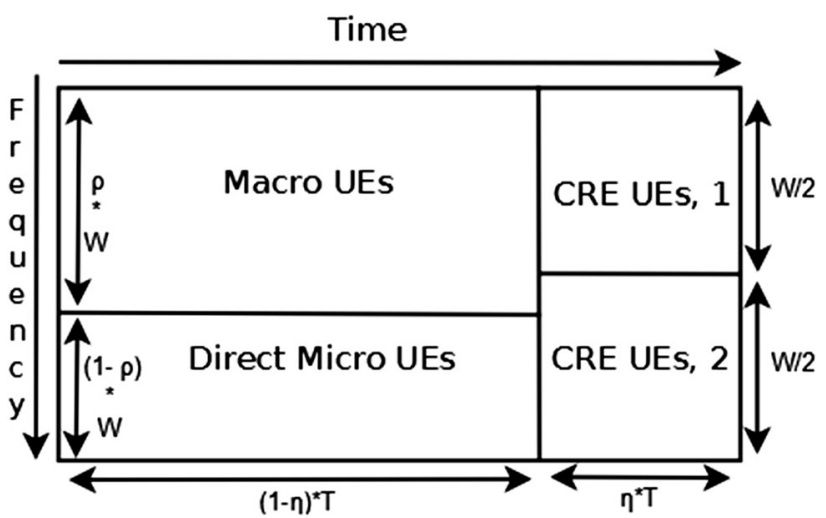

Fig. 2 Allocation of time and frequency to UEs

partitioning in the frequency domain. For CRE UEs, the whole band is divided into two equal parts for different CRE user groups, namely CRE UEs, 1 and CRE UEs, 2 . CRE UEs, 1 represent the users that are served by $B S_{i}$ with $i=1,3,5, \ldots, 2 n-1$, whereas CRE UEs, 2 are the users served by $B S_{i}$ with $i=2,4,6, \ldots, 2 n$. The reason behind partitioning the band for CRE UEs is to avoid interference coming to CRE UEs from neighboring Micro BSs. Frequency band is orthogonally shared among Macro and Direct Micro UEs, so that the interference power at Direct Micro UEs and Macro UEs is minimized. In our scheme, $\rho W$ of the total system bandwidth $W$ is used by Macro UEs. The time/frequency resources that are given to UEs are shared equally among UEs connected to the same BS. For example, if a Micro BS has $N_{m}$ Direct Micro UEs, each user has access to a channel with a bandwidth of $\frac{(1-\rho) W}{N_{m}}$ for $1-\eta$ in one unit of time. We also assume that each UE uses the maximum capacity of the channel assuming Gaussian alphabet is transmitted.

Using the communication model described above, we investigate the CDFs of data rate per UE, spectral and energy efficiencies (SE and EE) for the downlink communication. CDFs obtained here are not applicable for the uplink communication since power adaptation and different resource sharing methods should be considered in that case. We analytically derive CDF of data rate per UE using a geometrical approach and verify our analytical results using extensive simulations. A similar approach is followed to derive the analytical distributions of SE and EE. We selected 10th percentile rate $\left(R_{10}\right)$, median and tenth percentile Spectral $\left(S E_{50}, S E_{10}\right)$ and Energy Efficiency $\left(E E_{50}, E E_{10}\right)$ as Key Performance Indicators (KPIs). Employing the analytical CDF expression obtained for rate, SE and EE optimal values of resource sharing parameters $(\eta, \rho)$ that maximize these KPIs are obtained. 


\section{Analytical derivation of cumulative distribution of user rate, spectral and energy efficiency}

In this section, first, the analytical formulas for cumulative distribution of data rate/user for the UEs in the heterogeneous network model will be derived by using a geometrical approach. This approach is valid when Macro and Micro BSs are located at fixed locations. We assume a Cell-On-Edge configuration with a fixed $N_{\text {MICRO }}$ value, however the extension of the geometric approach to general cases will be discussed in Sect. 4.2. After obtaining the distribution for data rate/user, distributions for SE and EE will be derived using a similar approach.

As described in Sect. 3, there are three types of UEs in the system, which are Macro, Direct Micro and CRE UEs. Each type of these users have different data rate distributions and for given $B, \eta, \rho$ values a general equation for data rate per $\zeta$ type of user is given as,

$C_{\zeta}(B, \eta, \rho)=\frac{\eta_{\zeta}}{N_{B S, \zeta}(B)} W \rho_{\zeta} \log _{2}\left(1+\frac{P_{r}}{\sigma_{N+I, \zeta}^{2}}\right)$.

In (5), $\eta_{\zeta}$ is the time sharing parameter for user type $\zeta$. $N_{B S, \zeta}(B)$ is the average number of type $\zeta$ users being served by the same BS for bias value $B, W$ is the total bandwidth used, $\rho_{\zeta}$ is the band sharing parameter for user type $\zeta . P_{r}$ is the power of signal received from the associated BS. $\sigma_{N+I, \zeta}^{2}$ is the variance of Interference + Noise term of user type $\zeta$, which is modeled as a Gaussian random variable. $\zeta, \eta_{\zeta}$ and $\rho_{\zeta}$ are given as,

$$
\begin{aligned}
& \zeta=\left\{\begin{array}{l}
0, \quad \text { For a Macro user } \\
1, \quad \text { For a Direct Micro user } \\
2, \quad \text { For a CRE user },
\end{array}\right. \\
& \eta_{\zeta}=\left\{\begin{array}{cc}
1-\eta, & \zeta=0,1 \\
\eta, & \zeta=2,
\end{array}\right. \\
& \rho_{\zeta}=\left\{\begin{array}{cc}
\rho, & \zeta=0 \\
1-\rho, & \zeta=1 \\
0.5, & \zeta=2
\end{array}\right.
\end{aligned}
$$

The parameters $\eta, \rho$ in (8) are time and band sharing parameters which are illustrated in the resource allocation scheme shown in Fig. 2. In this paper, after obtaining the analytical expression for the data rate distribution, we aim to find the optimum values of $\eta$ and $\rho$ in order to maximize the tenth percentile rate, $R_{10}$.

\subsection{Modeling interference + noise term}

Interference + Noise term is modeled as a zero-mean Gaussian random variable with variance $\sigma_{N+I, \zeta}^{2}$ which is the summation of noise power $\left(P_{\text {noise }, \zeta}(B)\right)$ and interference power $\left(\sigma_{I, \zeta}^{2}\right)$ for type $\zeta$ user and given bias value $B$. Due to the symmetry of the BS locations in the heterogeneous network model, $\sigma_{N+I, \zeta}^{2}$ is assumed to be same for all users of the same type.

The noise part of Interference + Noise is a Gaussian random variable with variance $P_{n o i s e, \zeta}(B)$ and is calculated as in (9).

$P_{\text {noise }, \zeta}(B)=10^{\left(P_{n}+10 \log \left(w_{\zeta}(B)\right)+N F_{U E}\right) / 10}$.

In (9), $w_{\zeta}(B)$ is the average bandwidth in $\mathrm{Hz}$ that is used by a UE of type $\zeta, P_{n}(\mathrm{dBm} / \mathrm{Hz})$ is the noise spectral density and $N F_{U E}$ is the noise figure (in $\mathrm{dBm}$ ) of UEs. $w_{\zeta}(B)$ is calculated by the division of the total bandwidth used by type $\zeta$ UEs to the average number of type $\zeta$ UEs associated with the same BS for a fixed value of $B$. The calculation of the variance of the interference for different type of UEs will be presented below.

Macro UEs By inspecting the resource allocation scheme shown in Fig. 2, it can be observed that there is no source of interference for Macro UEs, therefore

$\sigma_{I, 0}^{2}=0$.

Direct Micro UEs Sources of interference for Direct Micro UEs are all Micro BSs other than the one that is associated with the UE. We have assumed that the total Interference for these users can be modeled as a Gaussian random variable with variance given by

$\sigma_{I, 1}^{2}=\sum_{i=2}^{N_{\text {MICRO }}} \frac{P_{2}}{l_{i}^{\alpha_{2}}}$.

In (11), $l_{i}$ is the distance between 1 st and $i$ th BSs for $i=2,3, \ldots, N_{\text {MICRO }}$.

CRE UEs Sources of interference for CRE UEs are the micro base stations that use the same portion of the band. Therefore as stated in Sect. 3, CRE UEs served by an odd indexed micro base station are interfered by odd indexed micro base stations whereas CRE UEs served by an even indexed micro base station are interfered by even indexed micro base stations. Since number of even indexed and odd indexed micro base stations are equal, the total Interference coming from odd or even indexed micro base stations are also equal and can be modeled as a Gaussian random variable with variance 
$\sigma_{I, 2}^{2}=\sum_{i=2 n}^{N_{\text {MICRO }}} \frac{P_{2}}{l_{i}^{\alpha_{2}}}$ for $\mathrm{n}=1,2, \ldots \frac{\mathrm{N}_{\text {MICRO }}}{2}$

\subsection{Distribution of received power}

The distribution of received power, $P_{r}$, should be obtained in order to find the distribution of the data rate per user which is given by (5). Figure 3 shows the range extended coverage of Micro BS for $B>0$. In this figure, the Micro BS coverage with $B=0$ is the blue region whereas the extended coverage with $B>0$ is the yellow region. Both yellow and blue regions satisfy that $P_{r, \text { Micro }} 10^{\frac{B}{10}}>P_{r, \text { Macro }}$, where $P_{r, \text { Micro }}$ is the power received from the closest Micro $\mathrm{BS}, P_{r, \text { Macro }}$ is the received power from Macro BS and $B$ is the BIAS parameter.

Assuming Macro BS is located at point $(0,0)$ and Micro $\mathrm{BS}$ is located at $\left(d_{\text {Micro }}, 0\right)$ any point having coordinates $(x, y)$ on the contour of Cell Range Extended coverage region should satisfy

$\frac{P_{1}}{\left(x^{2}+y^{2}\right)^{\frac{\gamma_{1}}{2}}}=10^{\frac{B}{10}} \frac{P_{2}}{\left(\left(x-d_{\text {Micro }}\right)^{2}+y^{2}\right)^{\frac{\gamma_{2}}{2}}}$.

Equation (13) is numerically solved for a given value of $B$ and extended coverage of Micro BS is obtained. But, it can be seen that the coverage area of a Micro BS is not a perfect circle. In order to simplify the analytical calculations, these coverage regions are approximated by circles. More detailed information on how this approximation is made and how close it is to the actual coverage can be found in the Sect. 2 of the technical report given in [27].

Using this approximated model for the system, the cumulative distribution of the received power $\left(P_{r}\right)$ for different types of users is derived using geometrical area calculations. First, we obtain the distribution of the distance of a user to its serving BS. Then using the distance distributions, the distributions of received power and data rate are obtained. Due to the space considerations in the paper, here we give the derivation of the distance and received power distribution of only Macro UEs. The derivations for different type of users can be found in the reference technical report [27]. Using CDFs obtained for $P_{r}$ for different types of UEs, the data rate per any UE will be derived in Sect. 4.3.

\subsubsection{Distribution of received power for macro UEs}

Distribution of the received power for Macro UEs can be found by first calculating the distribution of the distance between Macro UEs and Macro BS. The Macro BS coverage region is modeled as a combination of differently shaped regions as illustrated in Fig. $4 \mathrm{a}, \mathrm{b}$ for different $B$ values. The CDF of the distance between Macro UEs and Macro BS is given by

$F_{D}(d)=P(D \leq d)=\frac{S(d)}{S_{M A C R O}(B)}$.

In (14), $S(d)$ is the region where Macro UEs within a distance $d$ to Macro BS may reside. The area of this region is obtained by calculating the area of intersection of the circle centered at Macro BS location with a radius $d$ $\left(d \leq R_{\max }\right)$, with approximated Macro coverage region. This region is colored to orange in Fig. $4 \mathrm{a}, \mathrm{b} . S_{M A C R O}(B)$ is the total coverage area of the Macro BS and is the union of orange and green colored regions in Fig. $4 \mathrm{a}, \mathrm{b}$ for two different $B$ values. For ease of calculations these regions

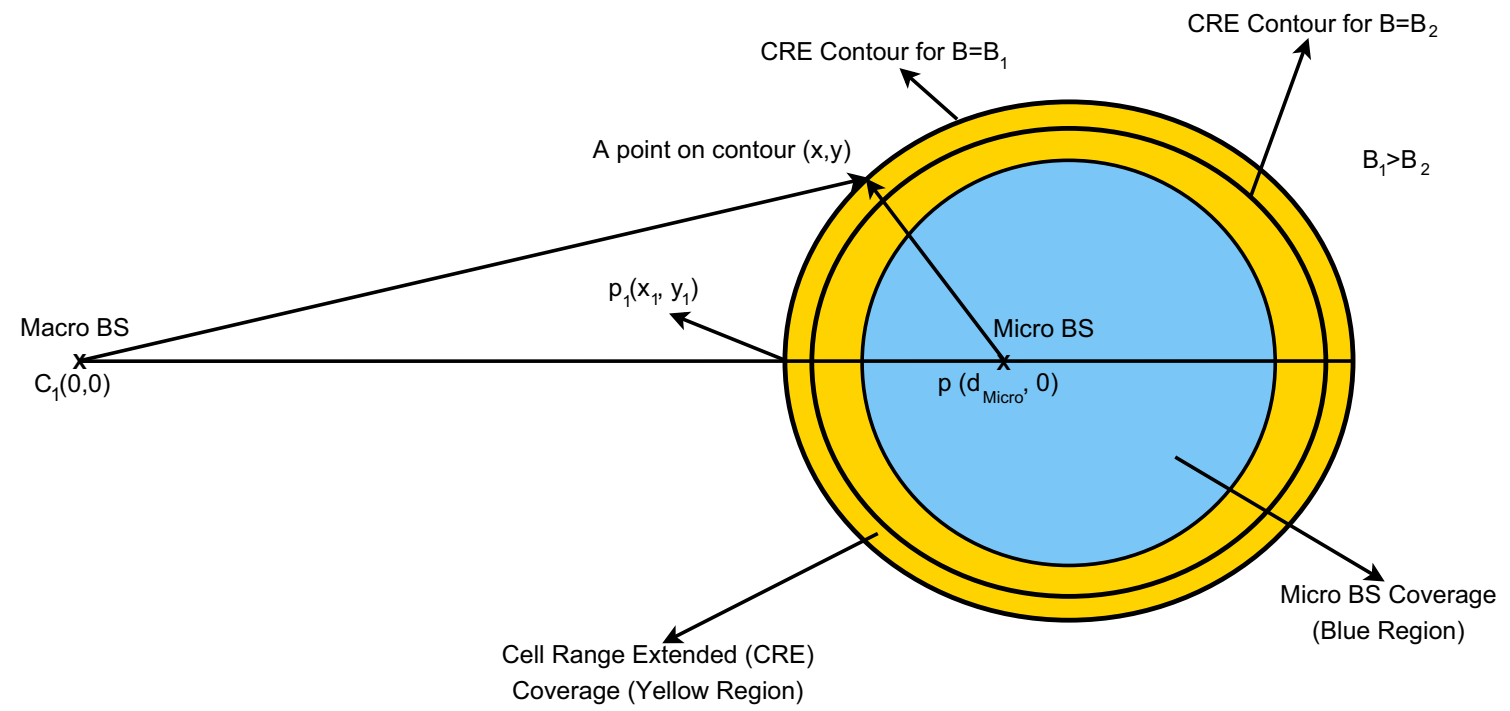

Fig. 3 CRE region contour for $B>0$ 


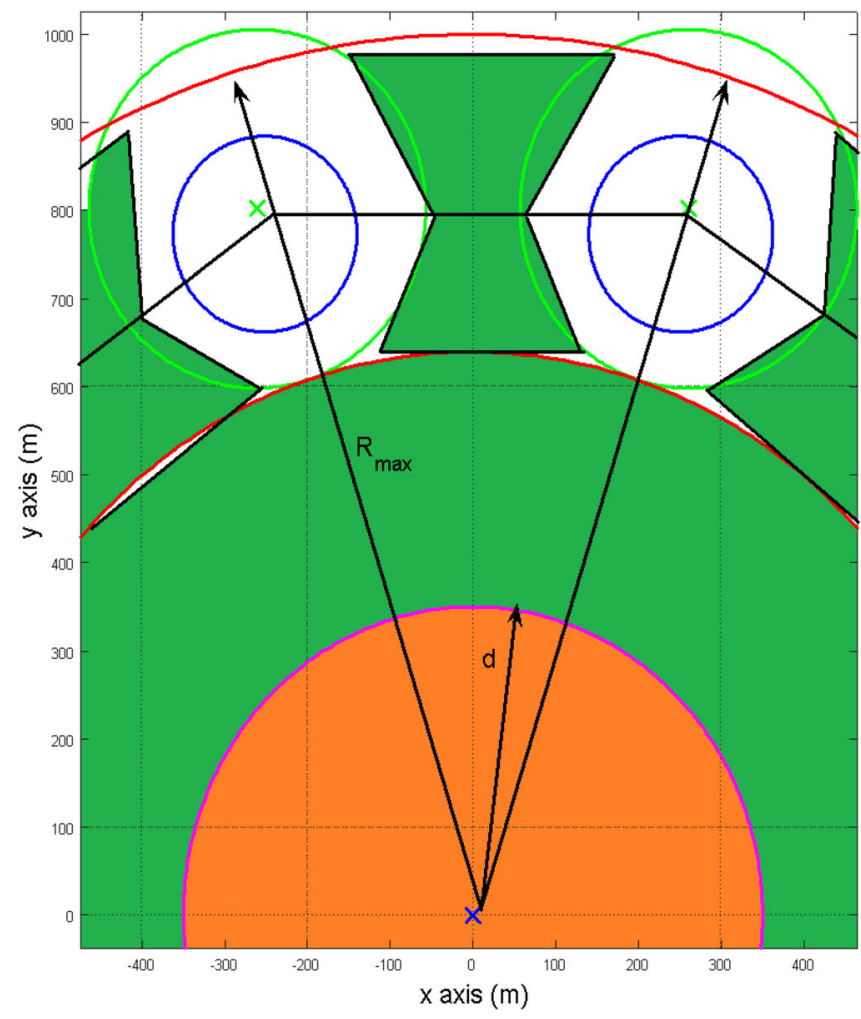

(a) $B=10 d B$

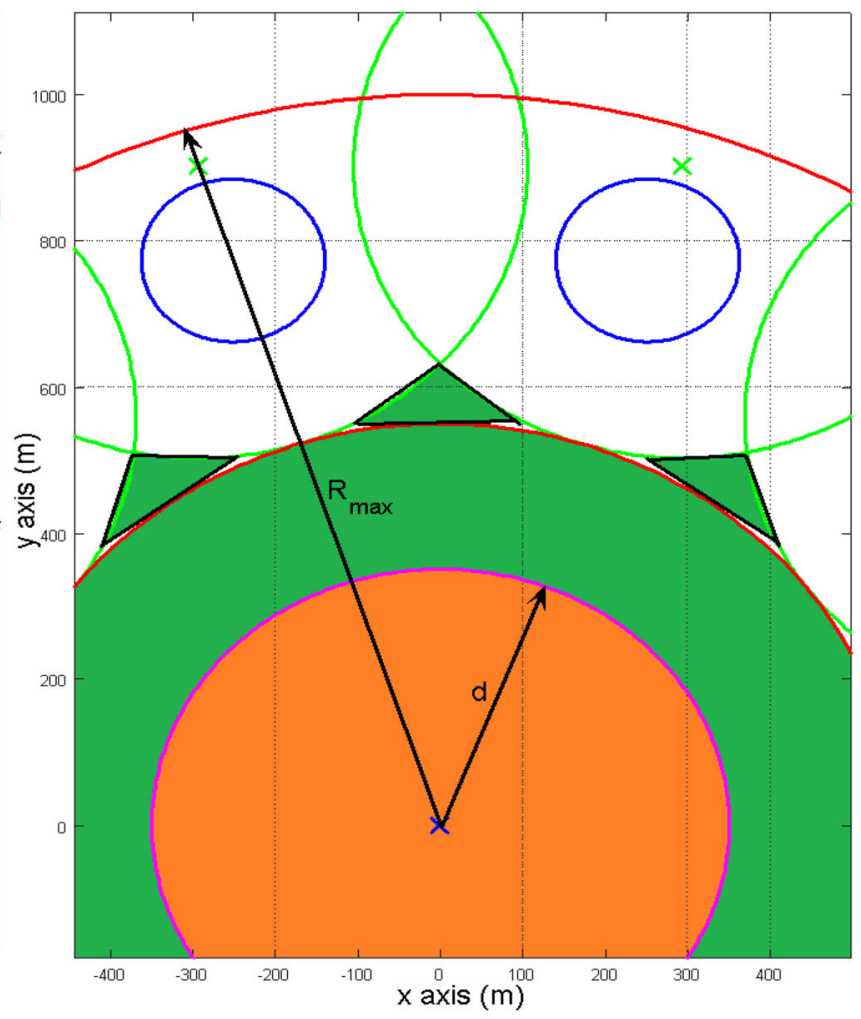

(b) $B=20 d B$

Fig. 4 Macro Region in detail for varying $B$ values

are approximated by 2D geometric shapes as triangle, trapezoid and circle.

Using CDF of $d$ given by (14) and the relation between $d$ and $P_{r}$ given by (1), the CDF of received power $P_{r}$ can be obtained as

$F_{p_{r}}\left(P_{r}\right)=1-F_{D}\left(\sqrt[-\alpha_{1}]{\frac{P_{r}}{P_{1}}}\right)$

\subsection{Distribution of data rate per user}

Using (5) and $F_{p_{r}}\left(P_{r}\right)$ for user type $\zeta$, the distribution of capacity for type $\zeta$ UEs is,

$F_{C}(c \mid \zeta, B, \eta, \rho)=F_{P r}\left(\sigma_{N+I, \zeta}^{2}\left(2^{\frac{c N_{B S \zeta \zeta}(B)}{n_{\zeta} W \rho_{\zeta}}}-1\right)\right)$.

Using (16) and CDFs of $P_{r}$ for different types of UEs (interested reader can refer to [27]), the distribution of the data rate per any UE in the network can be obtained as,
$F_{C}(c \mid B, \eta, \rho)=\sum_{\zeta=1}^{3} P(\zeta \mid B) F_{C}(c \mid \zeta, B, \eta, \rho)$,

where $P(\zeta \mid B)$ is the probability of being a type $\zeta$ user for a bias value of $B$ and is given by,

$P(\zeta=i \mid B)=\frac{N_{\zeta}(B)}{N_{U E}}, \quad \zeta=0,1,2$.

In (18), $N_{\zeta}(B)$ is the average number of UEs of type $\zeta$ for bias value $B$, and $N_{U E}$ is the total number of UEs in the system. According to the system model, a ratio of $1-$ $W_{\text {Micro }}$ of all UEs are distributed uniformly to all area, and a ratio of $W_{\text {Micro }}$ of UEs are distributed uniformly in Direct Micro coverage area. Using this model, $N_{\zeta}(B)$ is given by

$N_{\zeta}(B)=\left\{\begin{array}{cc}N_{U E}\left(1-W_{\text {micro }}\right) \frac{S_{M A C R O}(B)}{S_{T O T}}, & \zeta=0 \\ N_{U E} W_{\text {micro }}+N_{U E}\left(1-W_{\text {micro }}\right) \frac{S_{D I R}}{S_{T O T}}, & \zeta=1 . \\ N_{U E}\left(1-W_{\text {micro }}\right) \frac{S_{C R E}(B)}{S_{T O T}} & \zeta=2\end{array}\right.$ 
In Eq. (19) the areas given by $S_{M A C R O}(B), S_{D I R}, S_{C R E}(B)$ are the areas where Macro, Direct Micro, CRE users are located and $S_{T O T}$ is the whole cell area. The coverage areas of Macro and CRE users are calculated for the specific bias value of $B$. Coverage for Direct Micro UEs is independent of the bias value.

\subsection{Distribution of spectral efficiency and energy efficiency}

Spectral Efficiency (SE) is defined as the experienced data rate of a UE per bandwidth occupied by the UE. Energy Efficiency is the rate of UE divided by the total power consumed by the BSs of the system. SE and EE are expressed by,

$$
\begin{aligned}
& S E_{\zeta}(\eta)=\eta_{\zeta} \log _{2}\left(1+\frac{P_{r}}{\sigma_{N+I, \zeta}^{2}}\right), \\
& E E_{\zeta}(\eta)=\frac{C_{\zeta}(B, \eta, \rho)}{P_{t o t}(\eta)} .
\end{aligned}
$$

\subsubsection{Distribution of SE}

Distribution of $S E$ can be obtained similar to the data rate per UE distribution. Using the cumulative distribution of the received power $P_{r}$ for user type $\zeta$ and given time sharing parameter value $\eta$, the distribution of $S E$ can be obtained as

$$
F_{S E}(s \mid \zeta, B, \eta)=F_{P r}\left(\sigma_{N+I, \zeta}^{2}\left(2^{\frac{s}{n_{\zeta}}}-1\right)\right),
$$

By using (21), the CDF of $S E$ for any UE in the system can be written as

$F_{S E}(s \mid B, \eta)=\sum_{\zeta=1}^{3} P(\zeta \mid B) F_{S E}(s \mid \zeta, B, \eta)$,

where $P(\zeta \mid B)$ is the probability of being type $\zeta$ UE for a bias value of $B$.

\subsubsection{Distribution of EE}

In order to obtain the distribution of EE which is given by (20), $P_{\text {tot }}(\eta)$, total power consumed by BSs should be calculated. Here, we use a BS power consumption model proposed in [28], where the BS power consumption is modeled by a linear power model:

$$
P_{\text {in }}= \begin{cases}N_{\text {TRX }} P_{0}+\Delta_{p} P_{\text {out }}, & \text { if } 0<P_{\text {out }} \leq P_{\text {max }} \\ N_{\text {TRX }} P_{\text {sleep }}, & \text { if } \quad P_{\text {out }}=0\end{cases}
$$

where $P_{i n}$ is the total power consumed, $N_{T R X}$ is the number of transceivers in BS, $P_{0}$ is the power consumption at the
Table 1 Base station power consumption parameters

\begin{tabular}{lllll}
\hline BS type & $N_{T R X}$ & $P_{0}$ & $\Delta_{p}$ & $P_{\text {sleep }}$ \\
\hline Macro & 6 & 130 & 4.7 & 75 \\
Micro & 2 & 56 & 2.6 & 39 \\
\hline
\end{tabular}

minimum non-zero output power, $\Delta_{p}$ is the slope of the load-dependent power consumption, $P_{\text {out }}$ is the output power which is limited by $P_{\max }$ and $P_{\text {sleep }}$ is the sleep mode power consumption. The values of these parameters for Macro and Micro BSs are listed in Table 1.

By considering the model given by (23), the total power consumed by all $\mathrm{BSs}$ is given by

$$
\begin{aligned}
P_{\text {tot }}(\eta)= & (1-\eta) P_{\text {in }, 1}\left(P_{\text {out }}=P_{t, 1}\right) \\
& +\eta P_{\text {in }, 1}\left(P_{\text {out }}=0\right)+N_{\text {MICRO }} P_{\text {in }, 2}\left(P_{\text {out }}=P_{t, 2}\right) .
\end{aligned}
$$

In (24), $P_{\text {in }, 1}\left(P_{\text {out }}=P_{t, 1}\right)$ is the total power consumed by Macro BS when the output power is $P_{t, 1}$ and similarly $P_{i n, 2}\left(P_{\text {out }}=P_{t, 2}\right)$ is the total power consumed by a Micro BS when the output power is set to be $P_{t, 2}$. Using the distribution of the received power per UE, as given by (16), the distribution of $E E$ can be derived as

$$
F_{E E}(e \mid \zeta, B, \eta, \rho)=F_{P r}\left(\sigma_{N+I, \zeta}^{2}\left(2^{\frac{e P_{t o t}(\eta) N_{B S, \zeta}(B)}{\eta_{\zeta} P_{\zeta \zeta}}}-1\right)\right) \text {. }
$$

By using (25), the CDF of $E E$ for any UE in the network can be expressed as

$F_{E E}(e \mid \eta)=\sum_{\zeta=1}^{3} P(\zeta \mid B) F_{E E}(e \mid \zeta, B, \eta, \rho)$.

\subsection{Distribution of data rate per user, SE and EE for general cases}

The CDF of the data rate per user, $S E$ and $E E$ can also be obtained for different scenarios where small cells are placed at arbitrary locations. In order to obtain these distributions for general HetNet scenarios where BS locations are fixed, a similar geometric approach that we have followed for COE scenario can be used. By using the approximation used in Sect. 4.2, the coverage of small cell BSs can be modeled as circles. Using this approximation and geometric intersection formulas, the distance distribution of users can be obtained from which it is trivial to obtain distributions of received power, data rate, SE and EE. As an example, Fig. 5 shows a HetNet scenario in which small cell BSs are located at points which have 
Fig. 5 HetNet With two micro and one macro BS

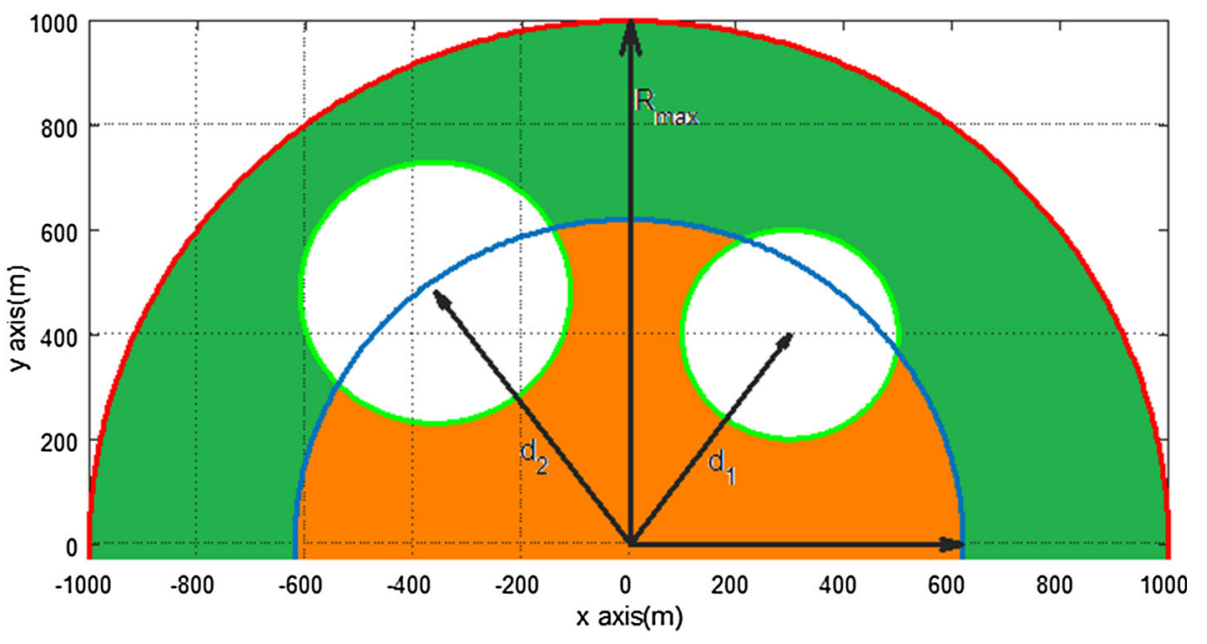

distances of $d_{1}$ and $d_{2}$ to Macro BS, respectively. In that scenario, using (14), the distribution of received power for a Macro Cell user can be obtained using the intersection area calculations as shown in Fig. 5. In Fig. 5, $S(d)$ is the orange colored region for a specific $d$ value, and $S_{M A C R O}(B)$ is the area of orange and green colored regions.

\section{Numerical results}

In this section, firstly analytical results obtained for the cumulative distribution of rate per UE will be compared with the rate distributions obtained from simulations. The comparisons are done for different bias $(B)$ and resource allocation parameter $(\eta, \rho)$ values and also for different UE distributions. Then, the analytical rate distribution is employed in order to optimize system parameters $\eta, \rho$ and $B$ for different UE distributions. The optimizations are done by considering tenth percentile rate $R_{10}$, which is the

Table 2 Parameter values

\begin{tabular}{ll}
\hline Parameter & Value \\
\hline$P_{t, \text { Macro }}$ & $46 \mathrm{dBm}$ \\
$P_{t, \text { Micro }}$ & $26 \mathrm{dBm}$ \\
$P_{\text {noise }}$ & $-173 \mathrm{dBm} / \mathrm{Hz}$ \\
BSNoiseFigure & $37 \mathrm{dBm}$ \\
$W$ & $100 \mathrm{MHz}$ \\
$\eta, \rho$ & $0 \leq \eta, \rho \leq 1$ \\
$\alpha_{1}$ & 3.5 \\
$\alpha_{2}$ & 4 \\
$N_{U E}$ & 1000 \\
$N_{\text {MICRO }}$ & 10 \\
$N_{\text {MACRO }}$ & 1 \\
\hline
\end{tabular}

parameter we have selected as KPI in the system. Optimization of $R_{10}$ is also done using simulations for comparison purposes.

We investigate the system in terms of Energy and Spectral efficiency. By using the $\rho$ values that maximizes $R_{10}$, we obtain the variation of tenth percentile and median of EE and SE by both using analytical expression and doing simulations. The analytical and simulation results are obtained with system model parameter values that are listed in Table 2.

The cumulative probability distribution of rate per UE obtained by analytical formula and simulations are plotted in Fig. 6. The CDFs are obtained for $\eta=0.2, \rho=0.5$, $B=10 \mathrm{~dB}$ and $B=20 \mathrm{~dB}$ and also for different UE distributions: $W_{\text {micro }}=\frac{1}{3}, \frac{1}{2}, \frac{2}{3}$. The goodness of fit between CDFs obtained by the analytical model and simulations are compared by using the Kolmogorov-Smirnov (KS) test [29]. Given an analytical distribution, this test shows whether random variables obtained empirically are distributed with the given analytical distribution or not for a given level of significance. In order to test the CDF obtained by analytical approximation, 100 UEs among 1000 UEs are randomly selected and the average level of significance between empirical and analytical distribution is calculated for 400 trials. Table 3 shows the ratio of the KS tests passed for a significance value of 0.05 , which is a typical significance value for KS test. Table 3 and Fig. 6 show us that the CDFs obtained by analytical approximation and simulations are very close to each other. Consequently, we conclude that the derived analytical CDF can be used for optimization of the network in terms of $R_{10}$.

In order to find optimal values of system parameters, we plot the variation of $R_{10}$ with respect to $\rho$ and $\eta$ for two different bias values, $B=10 \mathrm{~dB}$ and $B=20 \mathrm{~dB}$ and for 3 different spatial UE distributions. The variation of $R_{10}$ when $W_{\text {micro }}=\frac{1}{2}$ is depicted in Fig. 7. Figure 8 shows the 


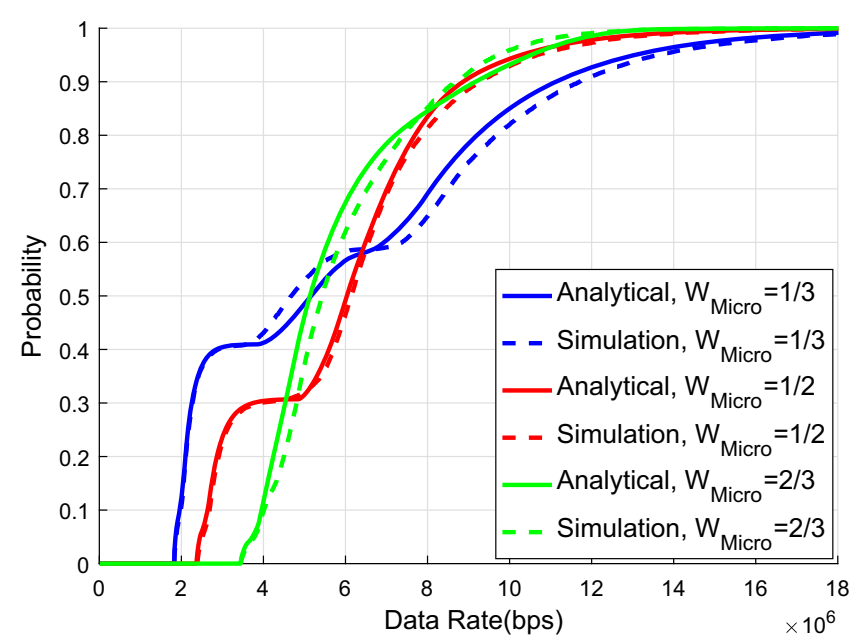

(a) $B=10 d B$

Fig. $6 \mathrm{CDF}$ of downlink data rate per user for $\eta=0.2, \rho=0.5$

Table $3 \%$ of $\mathrm{KS}$ tests passed for $\mathrm{CDF}$ of $R,\left(P_{\text {sig }}=5 \%\right)$

\begin{tabular}{llll}
\hline$B(\mathrm{~dB})$ & $W_{\text {Micro }}$ & & \\
\cline { 2 - 4 } & $1 / 3$ & $1 / 2$ & $2 / 3$ \\
\hline 10 & 0.8783 & 0.9033 & 0.5400 \\
20 & 0.9117 & 0.7650 & 0.6867 \\
\hline
\end{tabular}

cross-sections of variation of $R_{10}$ with respect to $\rho$ and $\eta$ for different spatial UE distributions. The cross-sections are plotted for $\rho$ values that maximize $R_{10}$. Table 4 show the

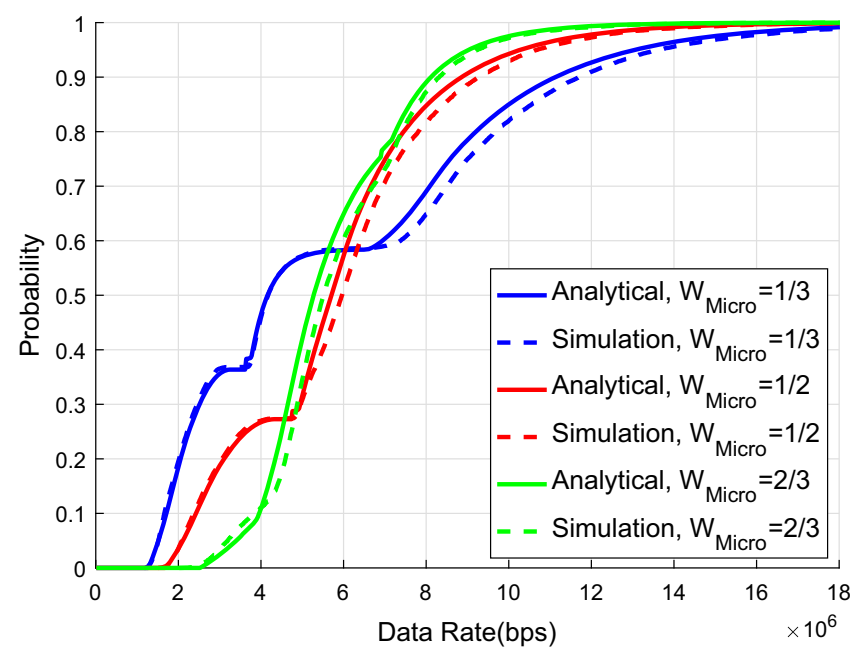

(b) $B=20 d B$

optimal $\eta$ and $\rho$ values and maximum $R_{10}$ values that are obtained from simulations and using the analytical CDF expressions for different UE distributions. By examining Fig. 8 and Table 4, it is observed that analytically obtained results are very close to simulation results. The optimal $\rho$ value decreases and optimal $\eta$ value increases with increasing values of $B$. Optimal $\rho$ value also decreases with increasing $W_{\text {micro }}$, which says that as the number of direct Micro UEs increases, larger portion of the bandwidth should be given to Micro BSs compared to small $W_{\text {micro }}$ values. Table 5 shows the results of KS test when it is applied to the distributions of SE and EE. Examining these

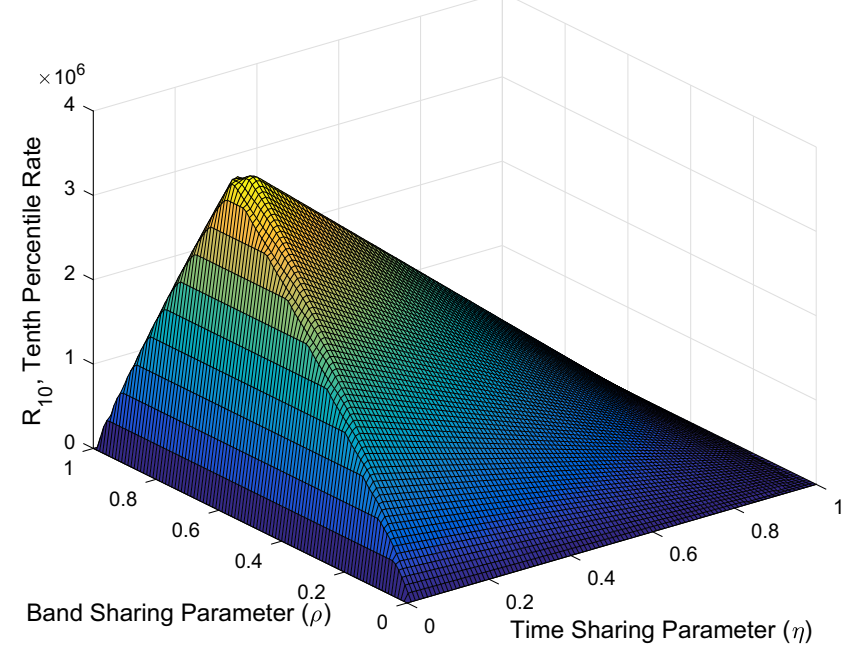

(a) $B=10 d B$

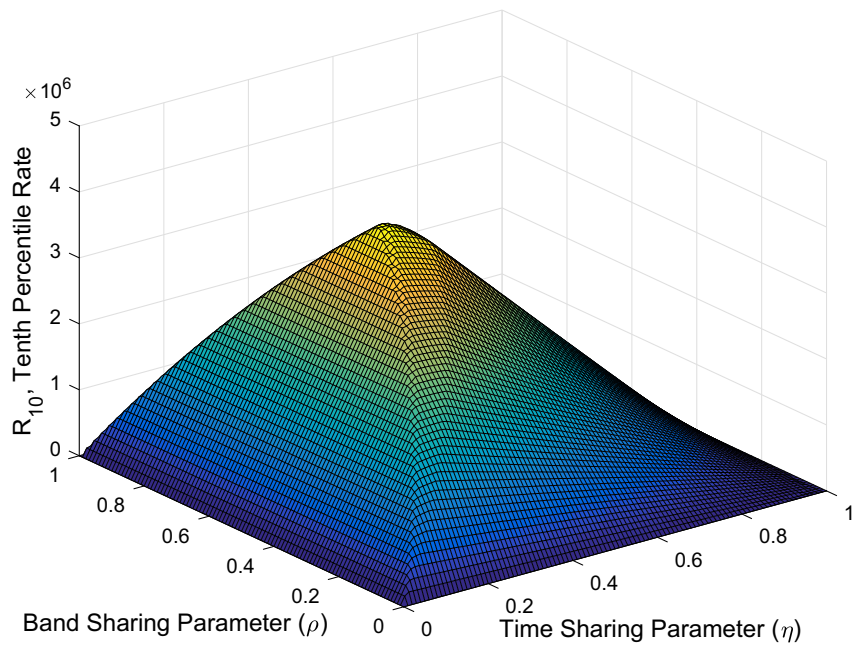

(b) $B=20 d B$

Fig. 7 Variation of $R_{10}$ as a function of $\rho$ and $\eta$ with $W_{\text {Micro }}=1 / 2$ 


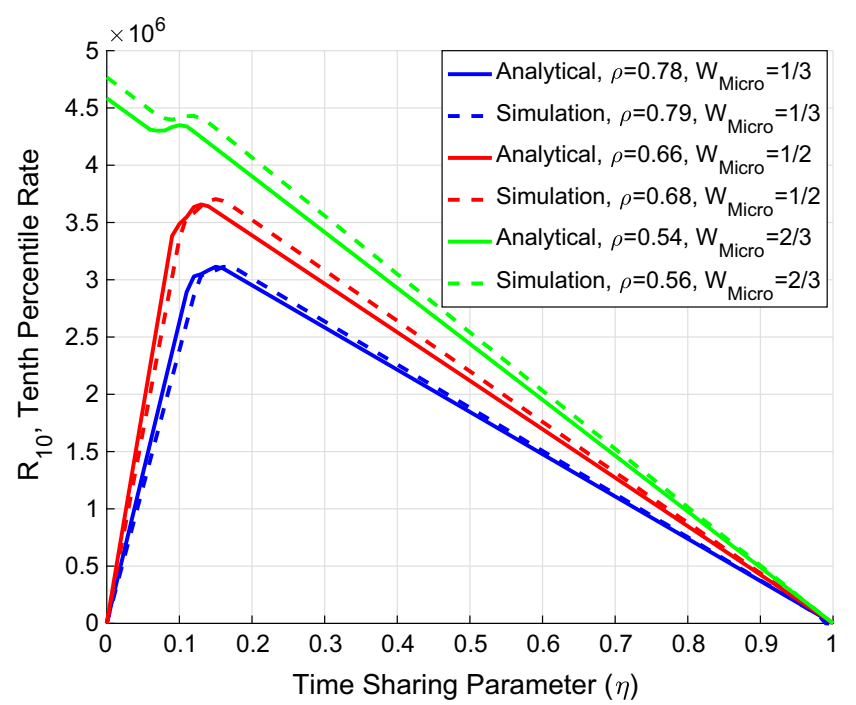

(a) $B=10 \mathrm{~d} B$

Fig. 8 10th Percentile downlink data rate for varying $\eta$ and $W_{\text {Micro }}$

Table 4 The comparison of optimal parameter values and $R_{10}$

\begin{tabular}{|c|c|c|c|c|c|c|}
\hline$W_{\text {micro }}$ & $\eta(\mathrm{S})$ & $\eta(\mathrm{A})$ & $\rho(\mathrm{S})$ & $\rho(\mathrm{A})$ & $R_{10}(\mathrm{~S})$ & $R_{10}(\mathrm{~A})$ \\
\hline \multicolumn{7}{|c|}{ (a) $B=10 \mathrm{~dB}$} \\
\hline $1 / 3$ & 0.17 & 0.15 & 0.79 & 0.78 & 3.115 & 3.113 \\
\hline $1 / 2$ & 0.15 & 0.13 & 0.68 & 0.66 & 3.704 & 3.657 \\
\hline $2 / 3$ & 0 & 0 & 0.56 & 0.54 & 4.768 & 4.585 \\
\hline \multicolumn{7}{|c|}{ (b) $B=20 \mathrm{~dB}$} \\
\hline $1 / 3$ & 0.43 & 0.41 & 0.67 & 0.66 & 3.433 & 3.416 \\
\hline $1 / 2$ & 0.36 & 0.35 & 0.53 & 0.50 & 4.095 & 4.011 \\
\hline $2 / 3$ & 0.27 & 0.25 & 0.38 & 0.35 & 4.799 & 4.786 \\
\hline
\end{tabular}

Table $5 \%$ of KS tests passed for CDFs of $S E$ and $E E,\left(P_{\text {sig }}=5 \%\right)$

\begin{tabular}{|c|c|c|c|c|c|c|}
\hline \multirow[t]{3}{*}{$B(\mathrm{~dB})$} & \multicolumn{3}{|l|}{ SE } & \multicolumn{3}{|l|}{$\mathrm{EE}$} \\
\hline & \multicolumn{6}{|l|}{$W_{\text {Micro }}$} \\
\hline & $1 / 3$ & $1 / 2$ & $2 / 3$ & $1 / 3$ & $1 / 2$ & $2 / 3$ \\
\hline 10 & 0.9233 & 0.8350 & 0.6683 & 0.9117 & 0.8383 & 0.5400 \\
\hline 20 & 0.8967 & 0.7950 & 0.6733 & 0.9067 & 0.7600 & 0.7000 \\
\hline
\end{tabular}

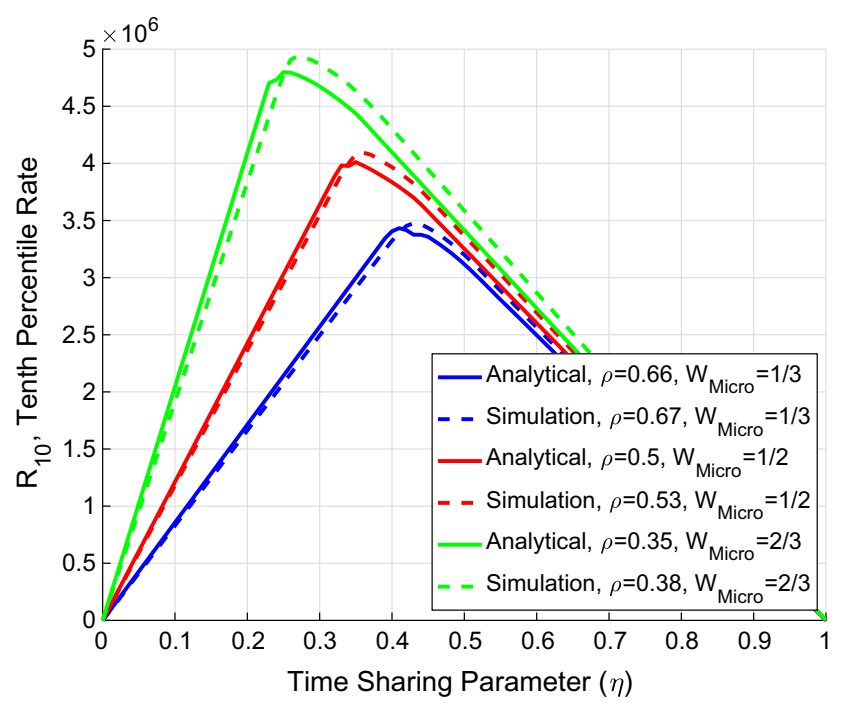

(b) $B=20 d B$

results, it can be concluded that the analytical distributions obtained can be used for further optimization of the system in terms of SE and EE. By examining Tables 3, 4 and 5, it can be concluded that the accuracy of the analytical model generally decreases with increasing $W_{\text {Micro }}$ and $B$ values. The reason behind this is the approximations done to simplify the base station coverage models get less accurate as $W_{\text {Micro }}$ and $B$ increases.

Figure $9 \mathrm{a}, \mathrm{b}$ show the variation of tenth percentile and median of $S E, E E$ with varying $\eta$ and $B$ values, respectively. It can be observed that median SE and EE decay nearly linearly as $\eta$ increases. Most of the UEs in the system is either Macro or Direct Micro UEs, therefore decreasing their resources also decrease the median SE and EE. However, this is not the case when $S E_{10}$ and $E E_{10}$ are considered. $S E_{10}$ and $E E_{10}$ are maximized at different values of $\eta$. When $E E_{10}$ is considered, the $\eta$ value which maximizes $E E_{10}$ is very close to the value that is optimal for $R_{10}$. This is because of the fact that variation of $R_{10}$ with respect to $\eta$ is faster compared to the variation of consumed power, $P_{t o t}$. Therefore, variation of $R_{10}$ dominates the variation of $E E$. If we analyze the system in terms of $S E_{10}$, it can be seen that, the $\eta$ value which maximizes $S E_{10}$ is different than that of $E E_{10}$. This result also exhibits the SE and EE trade-off in the Heterogeneous Network model as 

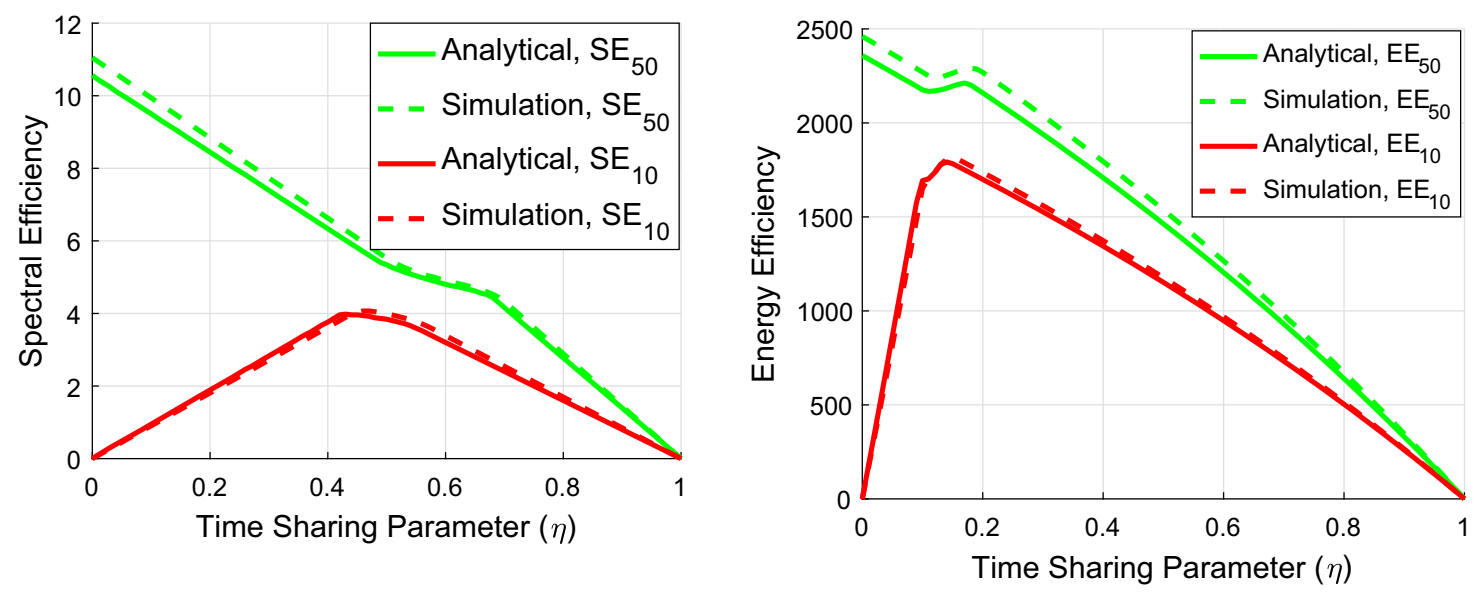

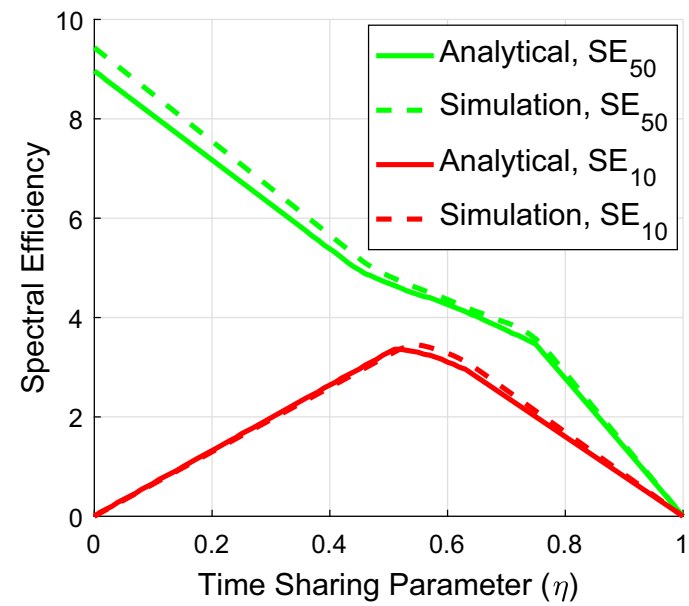

(a)

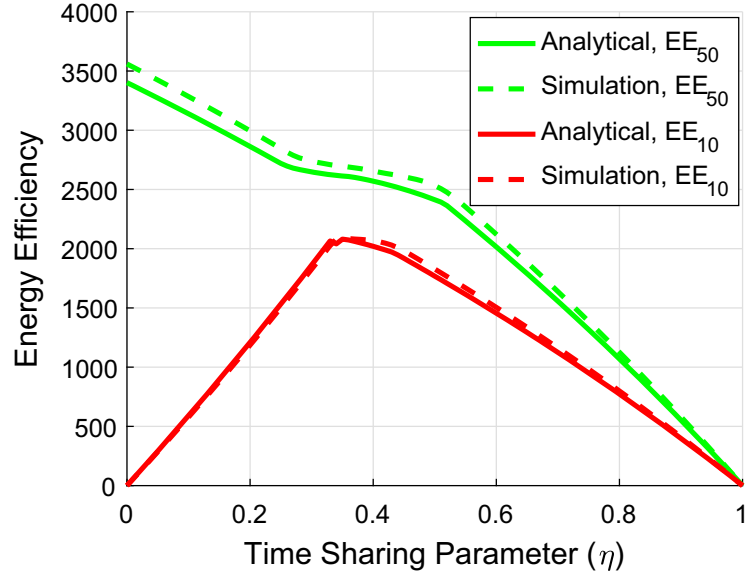

(b)

Fig. 9 Variation of $S E, E E$ as a function of $\eta$ for $\rho=0.5, B=10 \mathrm{~dB}$ (top), $B=20 \mathrm{~dB}$ (bottom) and $W_{\text {Micro }}=1 / 2$

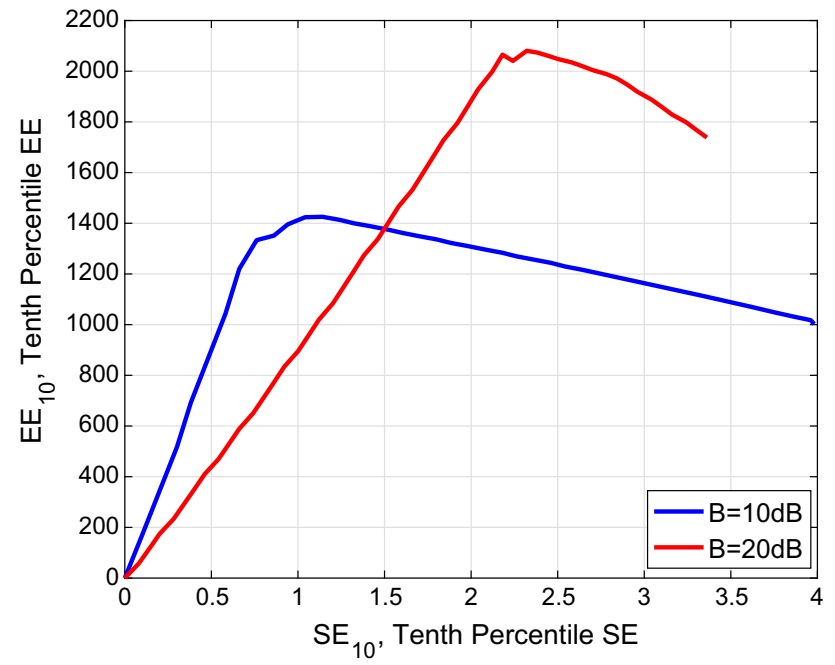

Fig. $10 S E_{10}$ versus $E E_{10}$ for $B=10 \mathrm{~dB}, 20 \mathrm{~dB}$ and $W_{\text {Micro }}=1 / 2$ shown in Fig. 10. By inspecting Fig. 9a, b it can also be concluded that the $\eta$ values maximizing $S E_{10}$ and $E E_{10}$ increase with $B$.

\section{Conclusion}

In this paper, we have analyzed a Heterogeneous Network with cell-edge located small cells. In the system, there is one Macro and $N_{M I C R O}$ Micro BSs and there are three types of UEs which are Macro, Direct Micro and CRE UEs. Assuming that time/frequency resources are shared orthogonally among these 3 types of UEs, we have analytically obtained the CDFs of user data rate, SE and EE by using a geometrical approach. We have shown that the analytically obtained CDFs are very close with the ones obtained from extensive simulations. We have used the analytical CDFs to optimize the system resource allocation 
parameters $\eta, \rho$ to maximize the Key Performance Indicators, such as $R_{10}, E E_{10}$ and $S E_{10}$. Our results show that the system is optimized around nearly same resource allocation parameter values when $R_{10}$ and $E E_{10}$ are considered. However, larger $\eta$ values are needed to maximize $S E_{10}$, where $R_{10}$ and $E E_{10}$ values degrade. This demonstrates the Energy Efficiency and Spectral Efficiency tradeoff in the HetNet system under consideration.

\section{References}

1. Li, Q. C., Niu, H., Papathanassiou, A. T., \& Wu, G. (2014). 5G network capacity: Key elements and technologies. IEEE Vehicular Technology Magazine, 9(1), 71-78.

2. Chin, W. H., Fan, Z., \& Haines, R. (2014). Emerging technologies and research challenges for $5 \mathrm{G}$ wireless networks. IEEE Wireless Communications, 21(2), 106-112.

3. Andrews, J. G., Buzzi, S., Choi, W., Hanly, S. V., Lozano, A., Soong, A. C. K., et al. (2014). What will 5G be? IEEE Journal on Selected Areas in Communications, 32(6), 1065-1082.

4. Boccardi, F., Heath, R. W., Lozano, A., Marzetta, T. L., \& Popovski, P. (2014). Five disruptive technology directions for 5G. IEEE Communications Magazine, 52(2), 74-80.

5. Damnjanovic, A., Montojo, J., Wei, Y., Ji, T., Luo, T., Vajapeyam, M., et al. (2011). A survey on 3GPP heterogeneous networks. IEEE Wireless Communications, 18(3), 10-21.

6. El-atty, S. M. A., \& Gharsseldien, Z. M. (2017). Performance analysis of an advanced heterogeneous mobile network architecture with multiple small cell layers. Wireless Networks, 23(4), 1169-1190.

7. 3rd Generation Partnership Project, "Scenarios and Requirements for Small Cell Enhancements for E-UTRA and E-UTRAN," In: 3GPP TR 36.932 V12.0.0, 2012.

8. Mishra, S., \& Murthy, C. S. R. (2018). Efficient coverage management of pico cells in hetnets via spectrum slicing, cell biasing, and transmit power spreading. Wireless Networks, 24(8), 3099-3112. https://doi.org/10.1007/s11276-017-1525-y.

9. 3rd Generation Partnership Project, "Summary of the Description of Candidate eICIC Solutions," In: 3GPP R1-104968, Madrid, Spain, 2010.

10. Fooladivanda, D., \& Rosenberg, C. (2013). Joint resource allocation and user association for heterogeneous wireless cellular networks. IEEE Transactions on Wireless Communications, 12(1), 248-257.

11. ElSawy, H., Sultan-Salem, A., Alouini, M. S., \& Win, M. Z. (2017). Modeling and analysis of cellular networks using stochastic geometry: A tutorial. IEEE Communications Surveys and Tutorials, 19(1), 167-203.

12. Renzo, M. D., Guidotti, A., \& Corazza, G. E. (2013). Average rate of downlink heterogeneous cellular networks over generalized fading channels: A stochastic geometry approach. IEEE Transactions on Communications, 61(7), 3050-3071.

13. Cheung, W. C., Quek, T. Q. S., \& Kountouris, M. (2012). Throughput optimization, spectrum allocation, and access control in two-tier femtocell networks. IEEE Journal on Selected Areas in Communications, 30(3), 561-574.

14. Heath, R. W., Kountouris, M., \& Bai, T. (2013). Modeling heterogeneous network interference using poisson point processes. IEEE Transactions on Signal Processing, 61(16), 4114-4126.
15. Mukherjee, S. (2012). Distribution of downlink SINR in heterogeneous cellular networks. IEEE Journal on Selected Areas in Communications, 30(3), 575-585.

16. Singh, S., Dhillon, H. S., \& Andrews, J. G. (2013). Offloading in heterogeneous networks: Modeling, analysis, and design insights. IEEE Transactions on Wireless Communications, 12(5), 2484-2497.

17. Singh, S., \& Andrews, J. G. (2014). Joint resource partitioning and offloading in heterogeneous cellular networks. IEEE Transactions on Wireless Communications, 13(2), 888-901.

18. Dhillon, H. S., Ganti, R. K., \& Andrews, J. G. (2013). Loadaware modeling and analysis of heterogeneous cellular networks. IEEE Transactions on Wireless Communications, 12(4), $1666-1677$.

19. Sambo, Y. A., Shakir, M. Z., Qaraqe, K. A., Serpedin, E., \& Imran, M. A. (2014). Expanding cellular coverage via cell-edge deployment in heterogeneous networks: spectral efficiency and backhaul power consumption perspectives. IEEE Communications Magazine, 52(6), 140-149.

20. Guvenc, I. (2011). Capacity and fairness analysis of heterogeneous networks with range expansion and interference coordination. IEEE Communications Letters, 15(10), 1084-1087.

21. Jung, T., Song, I., Lee, S., Jung, S., Yoon, S., \& Kang, J. (2018). Cell range expansion with geometric information of pico-cell in heterogeneous networks. In: IEEE 87th Vehicular Technology Conference (VTC Spring).

22. Lee, C.-N., Lin, J.-H., Wu, C.-F., Lee, M.-F., \& Yeh, F.-M. (2018). A dynamic CRE and ABS scheme for enhancing network capacity in LTE-advanced heterogeneous networks, Wireless Networks. https://doi.org/10.1007/s11276-018-1723-2.

23. Eguizábal, M., \& Hernández, A. (2013). Interference management and cell range expansion analysis for LTE picocell deployments. In: IEEE 24th Annual International Symposium on Personal, Indoor, and Mobile Radio Communications (PIMRC).

24. Teerasuttakorn, N., Nuanyai, K., Zamani, A., Schmeink, A., \& Chantaraskul, S. (2018). Study of almost blank subframe configurations for traffic offload in hetnets. In: International Conference on Information and Communication Technology Convergence (ICTC).

25. Jia, Y., Zhao, M., \& Zhou, W. (2016). Joint user association and eicic for max-min fairness in hetnets. IEEE Communications Letters, 20(3), 546-549.

26. 3rd Generation Partnership Project, Further advancements for E-UTRA physical layer aspects. In: $3 G P P$ TR $36.814 \quad 9.0 .0$ (2010).

27. Yenihayat, G., \& Karaşan, E. (2018). Derivation of users' received signal power in cell on edge configured hetnet communication system. In Technical report, Bilkent University, Ankara. http://kilyos.ee.bilkent.edu.tr/ guven/distanceDist. pdf. Accessed 5 Feb 2019.

28. Auer, G., Giannini, V., Desset, C., Godor, I., Skillermark, P., Olsson, M., et al. (2011). How much energy is needed to run a wireless network? IEEE Wireless Communications, 18(5), 40-49.

29. Panchenko, D. (2006). 18.443 statistics for applications, Massachusetts Institute of Technology: MIT OpenCourseWare. https://ocw.mit.edu/courses/mathematics/18-443-statistics-forapplications-fall-2003/lecture-notes/18443.pdf. License: Creative Commons BY-NC-SA, Fall. Accessed 17 Oct 2018. 


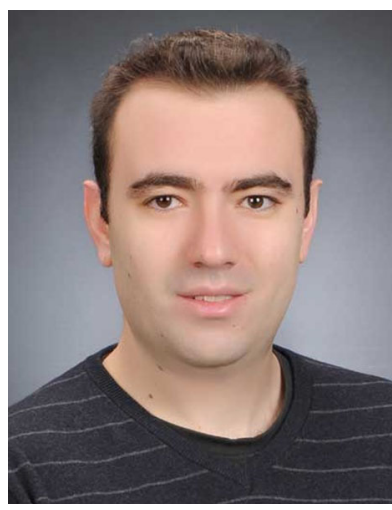

Güven Yenihayat received the B.S. and M.S. degrees in electrical and electronics engineering from the Middle East Technical University, Ankara, Turkey in 2008 and 2011, respectively. He is currently a $\mathrm{Ph} . \mathrm{D}$. candidate in the Department of Electrical and Electronics Engineering, Bilkent University, Ankara, Turkey. He also works as a Senior Design Engineer in ASELSAN, Turkey. His current research interests are next-generation wireless communications, heterogeneous networks and military ad hoc networks.

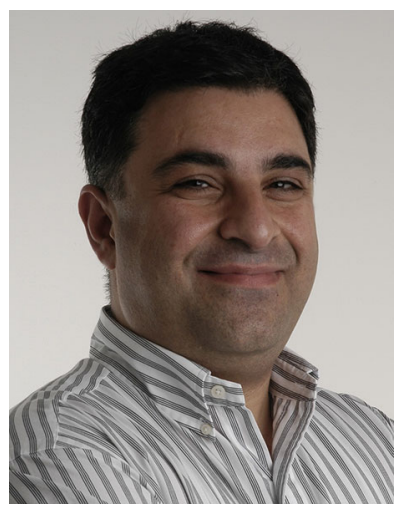

Ezhan Karaşan received the B.S. degree from Middle East Technical University, Ankara, Turkey, the M.S. degree from Bilkent University, Ankara, and the Ph.D. degree from Rutgers University, Piscataway, NJ, USA, in 1987, 1990, and 1995, respectively, all in electrical engineering. During 1995-1996, he was a Postdoctorate Researcher with Bell Labs, Holmdel, NJ. From 1996 to 1998, he was a Senior Technical Staff Member with the Lightwave Networks Research Department, AT\&T Labs Research, Red
Bank, NJ. Since 1998, he has been with the Department of Electrical and Electronics Engineering, Bilkent University, where he is currently a Full Professor. Since 2016, he has been the Dean of Faculty of Engineering and the Director of the Graduate School of Engineering and Science. He has participated in FP6-IST Network of Excellence (NoE) e-Photon/ONe+ and FP7-IST NoE BONE projects. His current research interests are in the application of optimization and performance analysis tools for the design, engineering, and analysis of optical and wireless networks. Dr. Karasan is a member of the Editorial Board of Optical Switching and Networking journal. He was a recipient of the 2004 Young Scientist Award from Turkish Scientific and Technical Research Council (TUBITAK), a Career Grant from TUBITAK in 2004, and the 2005 Young Scientist Award from Mustafa Parlar Foundation. He received a fellowship from the NATO Science Scholarship Program for overseas studies in 1991-1994.

Publisher's Note Springer Nature remains neutral with regard to jurisdictional claims in published maps and institutional affiliations. 